\title{
Representations of single and compound stimuli in negative and positive patterning
}

\author{
Justin A. Harris, Saba Gharaei, and Clinton A. Moore \\ University of Sydney, Sydney, New South Wales, Australia
}

\begin{abstract}
In four experiments, rats were trained on different patterning discriminations before being tested with compounds composed of novel combinations of the trained stimuli. In Experiment 1, rats were trained on a negativepatterning schedule $(\mathrm{A}+\mathrm{B}+\mathrm{AB}-)$ intermixed with reinforced presentations of a second compound $(\mathrm{CD}+)$. On a subsequent test, the rats responded more to two novel compounds, $\mathrm{AC}$ and $\mathrm{BD}$, than to $\mathrm{A}$ and $\mathrm{B}$, but less than to $\mathrm{CD}$. In Experiment 2, rats were trained on two concurrent negative-patterning discriminations $(\mathrm{A}+\mathrm{B}+$ $\mathrm{AB}-, \mathrm{C}+\mathrm{D}+\mathrm{CD}-)$. On test, they responded more to $\mathrm{AC}$ and $\mathrm{BD}$ than to $\mathrm{AB}$ and $\mathrm{CD}$, but less than to the single stimuli. In Experiment 3, rats were trained on two concurrent positive-patterning discriminations (A$\mathrm{B}-\mathrm{AB}+, \mathrm{C}-\mathrm{D}-\mathrm{CD}+)$. On test, their response rates to $\mathrm{AC}$ and $\mathrm{BD}$ were not different from the response rates to the trained compounds (AB and $\mathrm{CD}$ ). Finally, in Experiment 4, rats were trained on a positive- and negativepatterning discrimination concurrently. Once again, on test, response rates to $\mathrm{AC}$ and $\mathrm{BD}$ were not different from responding on reinforced trials of the trained discriminations $(\mathrm{A}+, \mathrm{B}+$, and $\mathrm{CD}+)$. We discuss the implications of these findings for elemental and configural models of stimulus representation.
\end{abstract}

Traditional theories of stimulus representation in associative learning have assumed that different stimuli are represented by distinct elemental units, or arrays of elemental units, and that, during conditioning, associations form between the elemental units of the conditioned stimulus (CS) and the unconditioned stimulus (US) (e.g., Bush \& Mosteller, 1951; Estes, 1950). Such theories also assume that conditioned responding is simply determined by the strength of the associations summed across individual CS elements (e.g., Rescorla \& Wagner, 1972). However, this second assumption has been challenged by demonstrations that animals can master negative-patterning discriminations, a task that explicitly reverses the reinforcement contingency between a simultaneous compound of two stimuli and the same stimuli presented individually. That is, each of two stimuli (A and B) is paired with the US $(+)$, but their compound is never followed by the US (i.e., the animals are exposed to intermixed trials of $\mathrm{A}+, \mathrm{B}+$, and $\mathrm{AB}-$ ). Animals have difficulty with this task but, nevertheless, learn to respond more to A and B than to AB (e.g., Pavlov, 1927; Rescorla, 1972; Whitlow \& Wagner, 1972). This is problematic for elemental theories if they assume that the associative strength of the $\mathrm{AB}$ compound is the linear sum of the strengths of the elements of A and B, because this assumption implies that responding to the compound should always exceed responding to A or B alone.

The fact that animals master negative-patterning discriminations shows that the representation of a compound is not necessarily the simple sum of its stimulus components. The conventional interpretation is that a compound of two or more stimuli is represented as the sum of its component stimuli plus a configural element that is activated uniquely by the compound (Spence, 1952). This allows the Rescorla-Wagner model to solve negative patterning, because the added configural element acquires strong inhibitory strength across $\mathrm{AB}$ - trials that cancels the combined excitatory strengths of the component stimuli (Rescorla, 1972; Whitlow \& Wagner, 1972).

To test this hypothesis, Rescorla (1972) and Whitlow and Wagner (1972) trained animals on a standard negativepatterning discrimination while concurrently conditioning a third stimulus (i.e., the animals received intermixed trials of $\mathrm{A}+, \mathrm{B}+, \mathrm{AB}-$, and $\mathrm{C}+$ ). Once they had mastered the discrimination, the animals were tested with two new compounds, AC and BC. The Rescorla-Wagner model predicts that responding to these compounds should be high because their component elements ( $\mathrm{A}$ and $\mathrm{C}$, or B and $\mathrm{C}$ ) would carry strong excitatory associative strength and, unlike responding to the $\mathrm{AB}$ compound, this would not be canceled by inhibition from any configural element. In both sets of studies, this prediction was confirmed: Responding was greater to $\mathrm{AC}$ and $\mathrm{BC}$ than to $\mathrm{AB}$ and, in one case (Whitlow \& Wagner, 1972), exceeded the level of responding elicited by the individual $\mathrm{A}, \mathrm{B}$, and $\mathrm{C}$ stimuli (a finding also consistent with the added configural element hypothesis).

There is evidence, however, that the added configural element hypothesis does not provide a comprehensive account of patterning discriminations under all circumstances (reviewed in Pearce, 1994; see also Williams,

J. A. Harris, justinh@psych.usyd.edu.au 
Gawel, Reimer, \& Mehta, 2005; Williams, Mehta, \& Dumont, 2004). In particular, if a redundant stimulus $(\mathrm{X})$ is added to a negative-patterning discrimination (i.e., training on an $\mathrm{AX}+\mathrm{BX}+\mathrm{ABX}-$ discrimination), the Rescorla-Wagner model predicts that this discrimination will be easier than the comparable discrimination without the redundant stimulus, whereas the available evidence indicates the opposite, that the redundant stimulus makes the discrimination harder (Pearce \& Redhead, 1993; Rescorla, 1972). Thus, the added configural element hypothesis does not provide an accurate description of learning of these more complicated patterning discriminations.

In the present article, we describe four experiments in which a Pavlovian magazine approach paradigm was used to investigate how rats solve "simple" patterning discriminations (i.e., between single CSs and two-stimulus compounds). The initial focus of these investigations was to test predictions derived from the Rescorla-Wagner model, given that this model does provide a satisfactory solution to such discriminations. The experiments followed the logic of the design used by Rescorla (1972) and Whitlow and Wagner (1972). In Experiment 1, rats were trained on a negative-patterning discrimination intermixed with reinforced presentations of a second compound $(\mathrm{A}+$, $\mathrm{B}+, \mathrm{AB}-, \mathrm{CD}+)$. They were then tested with these four stimulus configurations, as well as with two new compounds (AC and $\mathrm{BD})$ and the single stimuli (C and $\mathrm{D})$. In Experiment 2, rats were trained with two concurrent negative-patterning discriminations $(\mathrm{A}+, \mathrm{B}+, \mathrm{AB}-, \mathrm{C}+$, $\mathrm{D}+, \mathrm{CD}-)$. After training, the rats were tested with these six stimulus configurations, as well as with two new configurations ( $\mathrm{AC}$ and $\mathrm{BD}$ ). Experiment 3 used the same approach as Experiment 2, but in a positive-patterning discrimination that inverted the reinforcement contingencies of the negative-patterning design (this extended the present investigation of stimulus representation, because any mechanism proposed to operate during a negativepatterning schedule should also operate during positive patterning). Thus, the rats were trained with intermixed trials of $\mathrm{A}-, \mathrm{B}-, \mathrm{AB}+, \mathrm{C}-, \mathrm{D}-$, and $\mathrm{CD}+$ before being tested with these stimulus configurations and the two novel compounds (AC and BD). Finally, the rats in Experiment 4 were trained on concurrent negative- and positive-patterning discriminations $(\mathrm{A}+, \mathrm{B}+, \mathrm{AB}-, \mathrm{C}-$, $\mathrm{D}-, \mathrm{CD}+$ ) before being tested with those stimuli and the two novel compounds (AC and $\mathrm{BD}$ ). The results from each experiment will be compared with predictions derived from the added configural element hypothesis (Rescorla, 1972; Whitlow \& Wagner, 1972). In the General Discussion section of this article, we will consider the implications of all the results for several more recent elemental and configural models of stimulus representation (Harris, 2006; McLaren \& Mackintosh, 2002; Pearce, 1987, 1994; Wagner \& Brandon, 2001).

\section{EXPERIMENT 1}

In Experiment 1, rats were trained with a negativepatterning discrimination $(\mathrm{A}+, \mathrm{B}+, \mathrm{AB}-)$ intermixed with reinforced presentations of a second compound
$(\mathrm{CD}+)$. After extended training (60 days), they were tested with these four single and compound stimuli, as well as with two new compounds (AC and $\mathrm{BD}$ ) and the single stimuli (C and D; none of the new stimuli were reinforced during the test). This design is similar to that described by Rescorla (1972) and Whitlow and Wagner (1972), the key difference being that the rats were trained with a compound $(\mathrm{CD}+)$, rather than with a single, $\mathrm{CS}(\mathrm{C}+)$. The reason for this change was that the design of the earlier experiments $(\mathrm{A}+\mathrm{B}+\mathrm{AB}-\mathrm{C}+)$ creates a correlation between stimulus number (single vs. compound) and reinforcement, and if the rats learned this correlation (i.e., that compound trials are not reinforced), it would bias responding to the novel compounds on test. This might occur if the rats could use number or any property correlated with number (e.g., overall stimulation intensity) as a configural element that would readily generalize from $\mathrm{AB}$ to the test compounds. By training $\mathrm{CD}+$ rather than $\mathrm{C}+$, the present experiment removed this correlation, because $\mathrm{AB}-$ trials during training were balanced by $\mathrm{CD}+$ trials. The Rescorla-Wagner model predicts that responding to $\mathrm{AC}$ (or $\mathrm{BD}$ ) will be given by the summed associative strengths of A and C (or B and D). Because the associative strengths of $A$ and $B$ should be greater than those of $\mathrm{C}$ and $\mathrm{D}$, the $\mathrm{AC}$ and $\mathrm{BD}$ compounds should have greater associative strength and, thus, elicit more vigorous responding than should CD.

\section{Method}

\section{Subjects}

Sixteen experimentally naive male Hooded Wistar rats (Rattus norvegicus; weighing 280-357 g at the start of the experiment) were obtained from the breeding colony at the University of Adelaide, Australia, and were maintained in the animal colony in the School of Psychology at the University of Sydney. They were housed in groups of 8 in large white plastic tubs, measuring $26 \times 59 \times 37 \mathrm{~cm}$ (height $\times$ length $\times$ depth), with unrestricted access to water. Three days prior to commencement of the experiment, their access to food was restricted to $2 \mathrm{~h}$ per day (from 6 to 8 p.m.).

\section{Apparatus}

The rats were trained and tested in eight operant chambers measuring $28.5 \times 30 \times 25 \mathrm{~cm}$ (height $\times$ length $\times$ depth). The end walls of each chamber were made of aluminum; the sidewalls and ceiling were Plexiglas. The floor of the chamber consisted of stainless steel rods, $0.5 \mathrm{~cm}$ in diameter, spaced $1.5 \mathrm{~cm}$ apart. Each chamber had a recessed food magazine in the center of one end wall. A small metal cup measuring $3.5 \mathrm{~cm}$ in diameter and $0.5 \mathrm{~cm}$ deep was fixed on the floor of each food magazine. Attached to the food magazine was a dispenser delivering 45-mg food pellets (Noyes Formula P; Research Diets, New Brunswick, NJ). Each chamber was enclosed in a sound- and light-resistant wooden shell. Throughout all the sessions, fans located in the rear wall provided ventilation; the operation of these created a background level of noise measuring $70 \mathrm{~dB}$. Experimental events were controlled and recorded automatically by computers and relays located in the same room.

The stimuli were presented from four spatially separated sources. White noise (approximately $78 \mathrm{~dB}$ ) was presented from speakers mounted on the wall of each operant chamber above and to the right of the food magazine. A tone (approximately $78 \mathrm{~dB}$ and $2.9 \mathrm{kHz}$ ) was produced from a piezo buzzer positioned on the floor of the sound-attenuating shell behind each operant chamber. A flashing light $(2 \mathrm{~Hz})$ was produced by a $3 \times 5$ array of white LEDs, located on the floor of the sound-attenuating shell in front of the operant 
chamber. A steady light was produced by an incandescent bulb mounted high on the back wall of the sound-attenuating shell (the bulb was $100 \mathrm{~W}$ but was connected to a dimmer dial, so that the brightness was reduced to a level equivalent to that of a $15-\mathrm{W}$ bulb). Stimulus compounds consisted of the simultaneous presentation of two of the stimuli above from different modalities. The combination of different stimuli was counterbalanced across rats, with the constraint that all the compounds were composed of two stimuli from different modalities.

\section{Procedure}

Preexposure and magazine training. Before commencing training on the negative-patterning discriminations, the rats received context preexposure, stimulus preexposure, and magazine training across 3 consecutive days. On the 1st of these days, they were placed in their respective operant chambers for a period of $1 \mathrm{~h}$ and were permitted to freely explore without any stimuli or reinforcement. On the 2 nd day, they were placed in the chambers for $1 \mathrm{~h}$, during which each of the four stimuli was presented four times, intermixed in randomized order, without reinforcement. Stimulus presentations lasted for $30 \mathrm{sec}$ and were spaced on a 2.5-min (onset-to-onset) variable time (VT) schedule. On the 3rd day, all the rats received a 20 -min magazine training session, during which 20 food pellets were presented on a VT 1-min schedule, with no stimulus presentations.

Discrimination training (Days 1-60). The rats were trained with reinforced presentations of two single CSs (A and B; one auditory, one visual) intermixed with nonreinforced presentations of their compound (AB) and reinforced presentations of another audiovisual compound (CD). The specific allocation of the four stimuli was counterbalanced between subjects. On reinforced trials, presentation of the single or compound stimulus terminated with the delivery of a food pellet. On each of the first 25 days, each trial type was repeated six times. To facilitate mastery of the negativepatterning discrimination, on Days 26-32, the ratio of reinforced to nonreinforced trials was decreased, so that there were $4 \mathrm{~A}+, 4 \mathrm{~B}+$, and $4 \mathrm{CD}+$ trials, intermixed with $12 \mathrm{AB}-$ trials. This ratio was further decreased for Days 33-60: $3 \mathrm{~A}+, 3 \mathrm{~B}+$, and $3 \mathrm{CD}+$ trials were intermixed with $15 \mathrm{AB}-$ trials. Across all 60 days of training, the stimuli were presented on a VT 2.5-min schedule in a randomized order, with the constraint that no more than 2 trials of the same type occurred consecutively.

Test (Days 61 and 62). On both test days, the first 8 trials followed the training schedule $(1 \mathrm{~A}+, 1 \mathrm{~B}+, 1 \mathrm{CD}+$, and $5 \mathrm{AB}-$ trials). This was immediately followed by 24 test trials, comprising $2 \mathrm{~A}+, 2 \mathrm{~B}+, 4 \mathrm{CD}+, 4 \mathrm{AB}-, 4 \mathrm{AC}-, 4 \mathrm{BD}-, 2 \mathrm{C}-$, and $2 \mathrm{D}-$ trials. Trials of each type were randomly intermixed.

Across the entire experiment, the number and duration of photobeam interruptions by head entry into the magazine were recorded during each 30 -sec stimulus (single or compound) and the 30 -sec prestimulus interval. In this and the subsequent experiments, data are analyzed and presented showing the number of magazine entries during the $30-\mathrm{sec}$ stimulus minus the number in the preceding $30 \mathrm{sec}$. To ensure that the analysis of test performance included only data from rats that had adequately mastered the negative-patterning discrimination, we adopted a criterion that the rats' responding to $\mathrm{AB}$ must be greater than the average for $\mathrm{A}$ and $\mathrm{B}$ on at least 4 of the last 5 days of training. Analyses were conducted to confirm that, on tests, the rats continued to discriminate between the trained configurations and to confirm that responding to the single stimuli (C and D) was below responding to CD. However, the analyses of interest compared responding to the new compounds $(\mathrm{AC} / \mathrm{BD})$ with that to the trained compounds $(\mathrm{AB}$ and $\mathrm{CD})$ and with responding to the single stimuli $(\mathrm{A} / \mathrm{B} / \mathrm{C} / \mathrm{D})$. The latter comparison was done in two ways: (1) AC and $\mathrm{BD}$ were compared with the averages for $\mathrm{A}, \mathrm{B}, \mathrm{C}$, and $\mathrm{D}$; and (2) AC and BD were compared with whichever of their component stimuli elicited greater responding (e.g., they were compared with $\mathrm{A}$ and $\mathrm{B}$ if $\mathrm{A}>\mathrm{C}$ and $\mathrm{B}>\mathrm{D}$ ). These two comparisons tested different assumptions about the nature of summation between CSs in compound. The elemental approach of the Rescorla-Wagner model assumes that associative strengths of CSs combine additively in compound, and therefore, for summation to occur, responding to the compound must be greater than both individual CSs. But other models (e.g., Pearce, 1994) assume that the associative strength of a compound equals the average associative strength of the component CSs, and therefore, summation is observed if responding to the compound is greater than the average of responding to the individual CSs. The data from the final days of training were analyzed with paired $t$ tests to establish whether the rats had mastered the discriminations (i.e., were responding more on reinforced trials than on nonreinforced trials). The test data were subjected to an overall ANOVA, followed by direct contrasts comparing responding to the new compounds with that to the trained CSs and compounds.

\section{Results}

Responding to all the stimulus configurations increased over the first 25 days of discrimination training; the CD compound elicited the most responding, whereas the A and B CSs elicited the least. From that point, responding to the reinforced configurations $(\mathrm{A}, \mathrm{B}$, and $\mathrm{CD})$ remained stable, whereas responding to the nonreinforced compound (AB) slowly declined. For most rats, responding to $\mathrm{AB}$ eventually fell below the level elicited by $\mathrm{A}$ and $\mathrm{B}$ individually. However, 1 rat continued to respond more to $\mathrm{AB}$ than to $\mathrm{A}$ and $\mathrm{B}$, and a 2 nd rat did not appear to discriminate $\mathrm{AB}$ from $\mathrm{A}$ and $\mathrm{B}$. Thus, 14 rats reached criterion for inclusion in the subsequent data analysis (they responded less to $\mathrm{AB}$ than to the average of $\mathrm{A}$ and $\mathrm{B}$ on at least 4 of the last 5 days of training). Figure 1 shows the response rates of these 14 rats across the 60 days of discrimination training. It shows their average response rate during the single $\mathrm{CSs}$ ( $\mathrm{A}$ and $\mathrm{B}$ ) and the two trained compounds ( $\mathrm{AB}$ and $\mathrm{CD}$ ), minus their average baseline response rate (during the 30 -sec interval immediately before each stimulus presentation). By the end of training (across the final 5 days), the rats were responding significantly less to $\mathrm{AB}$ than to $\mathrm{A}$ and $\mathrm{B}[t(13)=5.80, p<.01]$ and were responding more to $\mathrm{CD}$ than to $\mathrm{A}$ and $\mathrm{B}[t(13)=$ $3.15, p<.01]$.

On Days 61 and 62, the rats were tested for responding to the trained stimulus configurations $(\mathrm{A}, \mathrm{B}, \mathrm{AB}$, and $\mathrm{CD})$, as well as two "new" compounds (AC and BD) and the single stimuli (C and D). Figure 1 shows these test data. An ANOVA revealed a significant overall effect $[F(4,52)=11.16, p<.01]$. Contrasts comparing responding to the new compounds with that to the trained CSs and compounds established that responding to the new compounds was significantly lower than responding to $\mathrm{CD}[F(1,13)=9.09, p=.01]$ but was significantly higher than responding to $\mathrm{AB}[F(1,13)=24.68, p<.01]$. Responding to the new compounds was also significantly greater than that to $\mathrm{C}$ and $\mathrm{D}[F(1,13)=8.21, p=.013]$ but was not significantly different from that to $\mathrm{A}$ and $\mathrm{B}$ $[F(1,13)=2.82, p=.117]$.

\section{Discussion}

After extensive training on a negative-patterning discrimination, rats responded less to a nonreinforced compound $(\mathrm{AB}-)$ than to the reinforced presentations of the single $\mathrm{CSs}(\mathrm{A}+$ and $\mathrm{B}+)$. They also responded most vigorously to a reinforced compound $(C D+)$ that was 

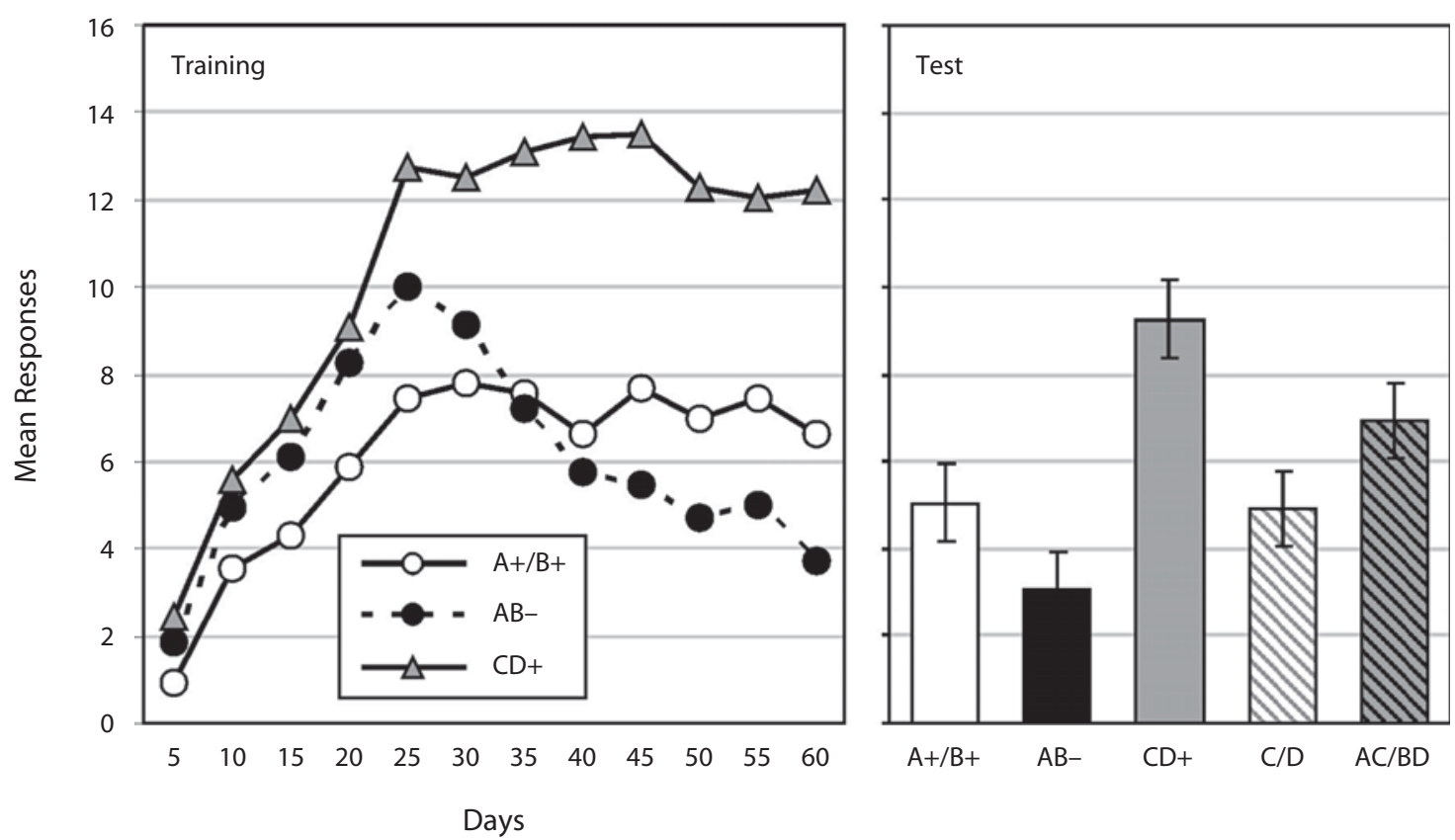

Figure 1. Left: Mean number of responses (magazine entries) above baseline during reinforced presentations of the single conditioned Stimuli $A$ and $B$, reinforced presentations of the $C D$ compound, and nonreinforced presentations of the $\mathrm{AB}$ compound, during training in Experiment 1. The data are presented in 5-day bins. Right: Mean number of responses to the trained stimulus configurations, as well as to the single stimuli $(C$ and $D)$ and two new compounds (AC and BD). Only data from the $\mathbf{1 4}$ rats that successfully acquired the patterning discrimination are plotted here.

intermixed among presentations of $\mathrm{A}, \mathrm{B}$, and $\mathrm{AB}$. The rats were then tested with presentations of two new compounds (AC and $\mathrm{BD})$ comprising one stimulus from each of the trained compounds. The rats responded less to these new compounds than to $\mathrm{CD}$, but more than to $\mathrm{AB}$. They responded more to $\mathrm{AC}$ and $\mathrm{BD}$ than to $\mathrm{C}$ and $\mathrm{D}$, but the difference between the new compounds and $\mathrm{A}$ and $\mathrm{B}$ was not significant.

The results on the final test can be accommodated by the added configural element hypothesis incorporated into the Rescorla-Wagner model (Rescorla, 1972; Whitlow \& Wagner, 1972). Responding to the new compounds ( $\mathrm{AC}$ and $\mathrm{BD}$ ) should be greater than responding to the individual CSs, as was partially observed, due to summation of associative strengths between stimuli in a compound. Moreover, the associative strength of these new compounds should be below that of the trained compound $\mathrm{CD}$ to the extent that the configural unit for $\mathrm{CD}$ acquired associative strength. That is, because the associative strength of CD should equal the associative strengths of $\mathrm{C}$ and $\mathrm{D}$ plus the associative strength of the $\mathrm{CD}$ configural element, and because the associative strengths of A, B, C, and $\mathrm{D}$ are all equal, the associative strength of $\mathrm{AC}$ should equal the associative strength of CD minus the associative strength of the CD configural unit.

There is one aspect to the data in the present experiment that poses difficulties for the Rescorla-Wagner model. The analysis presented at the end of the preceding paragraph is based on the assumption that conditioning to A and $\mathrm{B}$ had not reached asymptote by the end of conditioning, a conclusion potentially supported by the fact that respond- ing to those stimuli was well below response rates to the CD compound. However, an inspection of Figure 1 makes it clear that the response rates to $\mathrm{A}$ and $\mathrm{B}$ had not changed over the last half of the training (from Day 25 to Day 60). Responding to the CD compound also remained stable across this same period. This implies that conditioning to $\mathrm{A}$ and $\mathrm{B}$ and to $\mathrm{CD}$ had reached asymptote well before the end of training. The Rescorla-Wagner model predicts that conditioning to $\mathrm{A}$ and $\mathrm{B}$ should reach the same asymptote as that to $\mathrm{CD}$, a prediction that is obviously at odds with the evidence here that asymptotic responding to $\mathrm{A}$ and $\mathrm{B}$ was well below that to CD.

\section{EXPERIMENT 2}

In Experiment 2, rats were trained with two concurrent negative-patterning discriminations, in which they received intermixed trials of $\mathrm{A}+, \mathrm{B}+, \mathrm{AB}-, \mathrm{C}+, \mathrm{D}+$, and $\mathrm{CD}-$. They were then tested with these plus two new compounds (AC and BD). This constitutes a strict test of the difference between these new compounds and the trained compounds (AB and $\mathrm{CD}$ ), because all the compounds consist of stimuli with identical reinforcement histories. This is important because the associative strength of one stimulus in a compound may affect how the associative strength of the other stimulus is expressed in performance. For example, in Experiment 1, how Stimulus A's associative strength was expressed in responding may have differed between the $\mathrm{AB}$ and $\mathrm{AC}$ compounds by virtue of the difference in the associative histories of $\mathrm{B}$ and $\mathrm{C}$. By contrast, in the present experimental design, 
both stimuli in each new compound had been trained as part of a negative-patterning discrimination, and therefore the associative histories of all the stimuli were identical. Nonetheless, if rats solve negative-patterning discriminations by learning about the configural elements specific to the nonreinforced compounds (AB and $\mathrm{CD}$ ), this should not affect, or have less effect on, their responding to the new compounds. Therefore, the rats should respond strongly to the new compounds (AC and $\mathrm{BD}$ ).

The experiment was run as two replications. In the first replication, training on the two negative-patterning discriminations could proceed only for 35 days, at which point only 9 of the original 16 rats had mastered both discriminations (according to the criterion used in Experiment 1). To increase statistical power, the experiment was replicated with another 15 rats that were trained for 40 days, after which 10 had satisfactorily mastered both discriminations. This second replication was extended for 8 days beyond the tests, during which time the rats resumed negative-patterning training with one pair of stimuli $(\mathrm{A}+$ $\mathrm{B}+\mathrm{AB}-)$ but switched to positive-patterning training with the other set $(\mathrm{C}-\mathrm{D}-\mathrm{CD}+)$. This extension was conducted to test how effectively the rats distinguished between the two sets of stimuli.

Method
Subjects and Apparatus
Thirty-one experimentally naive Hooded Wistar rats ( 16 females
weighing $212-254 \mathrm{~g} ; 15$ males weighing $280-340 \mathrm{~g}$ ) were housed in
the animal colony in the School of Psychology at the University of
Sydney. The 16 females were bred in that colony; the 15 males were
obtained from the breeding colony at the University of Adelaide,
Australia. All the rats were housed in same-sex groups of 8 (or 7 )
in the same manner as in Experiment 1 . Three days prior to com-
mencement of the experiment, their access to food was restricted
to $2 \mathrm{~h}$ per day (from 3 to 5 p.m. or from 5 to 7 p.m.). The rats were
trained and tested in the same chambers, using the same stimuli as
those in Experiment 1.

\section{Procedure}

Training. The rats received context preexposure, stimulus preexposure, and magazine training across 3 consecutive days, as in Experiment 1. Following this, the rats received 5 days of reinforced training with the individual stimuli. Each stimulus was presented four times (16 trials in total) for $30 \mathrm{sec}$ on a VT 2.5 -min schedule, with the constraint that no more than 2 trials of the same type occurred consecutively. This was followed by 9 days of discrimination training with the two nonreinforced compounds (each comprised one auditory and one visual stimulus, counterbalanced): The rats received 4 presentations of each single stimulus and 8 presentations of each of the two compounds. For the next 20 days ( 25 days in the second replication), the ratio of nonreinforced to reinforced presentations was doubled to facilitate mastery of the discrimination. Thus, on each daily session, the rats received 3 reinforced presentations of each single stimulus and 12 nonreinforced presentations of each compound.

Test. The rats were tested on consecutive days. Before the first test, they completed a short training session, identical to the previous schedule but with fewer trials (i.e., there were two reinforced presentations of each single stimulus, and eight nonreinforced presentations of the compounds). This was followed by a test under extinction: The rats received two nonreinforced presentations of each of the four single stimuli (A, B, C, and D), plus four nonreinforced presentations of the previously trained compounds (AB and $C D)$, as well as four nonreinforced presentations of two new compounds
(AC and BD). Trials of each type were randomly intermixed. The second test, the following day, used the same reinforcement schedule as that used during training but included four nonreinforced presentations of the two new compounds.

In the second replication, the experiment was extended for 8 days beyond the test. During this period, one discrimination was changed from negative patterning to positive patterning (i.e., the previous schedule of $\mathrm{C}+\mathrm{D}+\mathrm{CD}$ - was changed to $\mathrm{C}-\mathrm{D}-\mathrm{CD}+$ ), whereas the negative-patterning schedule was maintained for the other pair of stimuli $(\mathrm{A}+\mathrm{B}+\mathrm{AB}-)$. The number of trials and intertrial spacing were the same as those used in the earlier training phases.

\section{Results}

Responding to all the stimulus configurations increased during the first 20 days of training. Over this period, the level of responding was very similar between the two replications $[F(1,29)=0.249, p=.62]$. However, after this point, the rats in the first replication displayed higher response rates than did the rats in the second replication. Averaged over the last 15 days of training, the difference between the two groups was significant $[F(1,29)=7.56$, $p=.01]$. Over this same period, the rats responded significantly more to the single CSs than to the compounds $[F(1,29)=54.08, p<.01]$, and, importantly, this difference did not interact with the replication factor $[F(1,29)=$ $0.20, p=.66]$. In other words, despite the difference in overall level of responding, the rats in the first and second replications showed equivalent discrimination performance. Therefore, given that all relevant analyses were within subjects, we combined the data from the two replications.

We were concerned that the test results should include only data from those rats that had learned both negativepatterning discriminations, and therefore, we applied the criterion used in Experiment 1 to both discriminations. That is, for at least 4 of the last 5 days of training, the rats must have responded less to $\mathrm{AB}$ than to the average of $\mathrm{A}$ and $\mathrm{B}$ and less to $\mathrm{CD}$ than to the average of $\mathrm{C}$ and $\mathrm{D}$. Nine rats reached this criterion in the first replication; 10 rats reached this criterion in the second replication. For these 19 rats, responding was, on average, $50 \%$ lower to the trained compounds than to the single CSs. Figure 2 shows their training data across the last 15 days of training.

After training, the rats were tested for responding to the single stimuli (A, B, C, and D), the trained compounds (AB and $\mathrm{CD}$ ), and two new compounds ( $\mathrm{AC}$ and $\mathrm{BD}$ ). Figure 2 shows these data. An ANOVA revealed a significant overall effect $[F(2,36)=13.98, p<.01]$. Contrasts comparing responding to the new compounds with that to the trained CSs and compounds established that the rats responded significantly less to the trained compounds than to the new compounds $[F(1,18)=15.64, p<.01]$. The rats also responded significantly less to the new compounds than to the single CSs $[F(1,18)=5.13, p=.036]$.

In the second replication, the experiment was extended: One discrimination was changed from negative patterning to positive patterning $(\mathrm{C}-\mathrm{D}-\mathrm{CD}+)$, and a negativepatterning schedule was maintained for the other pair of stimuli $(\mathrm{A}+\mathrm{B}+\mathrm{AB}-)$. The performance of the 10 rats that had reached criterion previously is shown in Figure 2 (the plots include responding on the final day, Day 40, of 

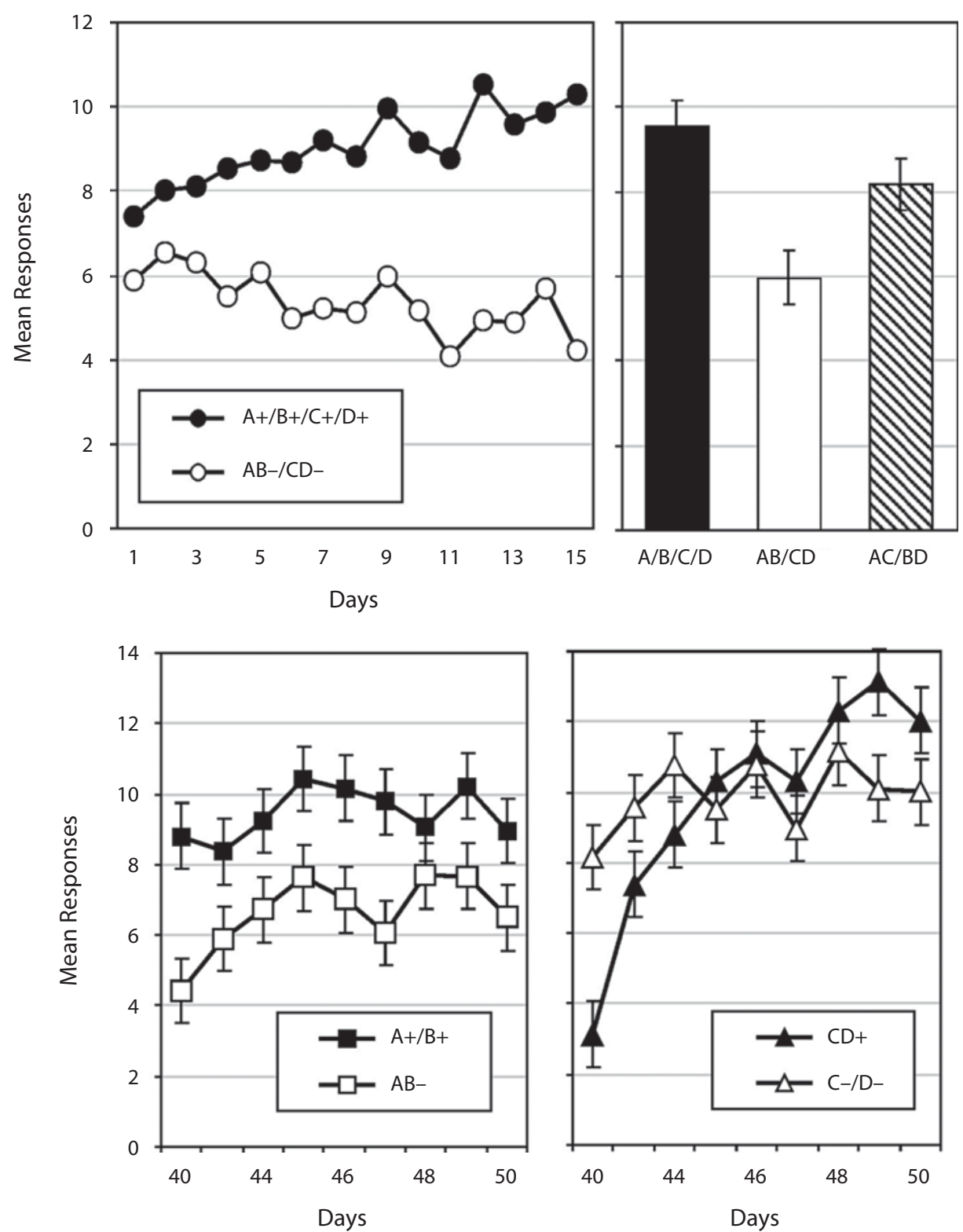

Figure 2. Top left: Mean number of responses (magazine entries) above baseline during reinforced presentations of single conditioned stimuli (A, B, C, and D) and nonreinforced presentations of compound stimuli ( $A B$ and $C D$ ) across the last 15 days of negative patterning training in Experiment 2. Top right: Mean number of responses to the same single and compound stimuli, as well as two new compounds (AC and BD) composed of novel combinations of the trained stimuli, on subsequent tests. Only data from the 19 rats that successfully acquired the patterning discrimination are plotted. Bottom row: Response rates for 10 rats given posttest retraining in which one pair of stimuli continued to be reinforced according to a negative-patterning schedule $(A+B+A B-$; on the left), whereas the other pair were switched to a positive-patterning schedule $(\mathrm{C}-\mathrm{D}-\mathrm{CD}+$; on the right).

the initial training). Clearly, reversal of the reinforcement schedule increased responding to $\mathrm{CD}$, so that the rats ultimately responded more to $\mathrm{CD}$ than to $\mathrm{C}$ and $\mathrm{D}$ individually. In contrast, these same rats continued to respond more to $\mathrm{A}$ and $\mathrm{B}$ than to $\mathrm{AB}$. This difference was confirmed by an ANOVA showing a significant three-way interaction between discrimination schedule (negative vs. positive patterning), stimulus configuration (compound vs. single stimuli), and linear trend across days $[F(1,9)=18.87, p<$
.01]. Simple comparisons confirmed the source of this interaction: Across the first 2 days of extended training (Days 43 and 44), the rats responded more to A and B than to $\mathrm{AB}[F(1,9)=7.56, p=.02]$ and, likewise, responded more to $\mathrm{C}$ and $\mathrm{D}$ than to $\mathrm{CD}[F(1,9)=11.09, p=.01]$, but by the last 2 days of this training (Days 49 and 50), they responded more to $\mathrm{CD}$ than to $\mathrm{C}$ and $\mathrm{D}[F(1,9)=7.73$, $p=.02]$, while still responding more to $\mathrm{A}$ and $\mathrm{B}$ than to $\mathrm{AB}[F(1,9)=8.64, p=.02]$. This demonstrates that the 
rats did distinguish between the two pairs of stimuli, so that they could learn opposite reinforcement schedules for $\mathrm{AB}$ versus $\mathrm{CD}$.

\section{Discussion}

In this experiment, rats were trained with two concurrent negative-patterning discriminations $(\mathrm{A}+\mathrm{B}+\mathrm{AB}-$ and $\mathrm{C}+\mathrm{D}+\mathrm{CD}-)$. When tested with new compounds $(\mathrm{AB}$ and $\mathrm{CD})$ formed by combining stimuli across the two discriminations, the rats responded more to these new compounds than to the trained compounds, but less than to the individual CSs. The added configural element hypothesis, framed within the Rescorla-Wagner model, correctly predicts that responding to the new compounds should be above the level elicited by the trained compounds. However, this model does not predict that rats should respond less to the new compounds than to the individual CSs. Rather, it incorrectly predicts that responding to the new compounds should exceed that shown to the single CSs.

We also asked whether the rats distinguished adequately between the stimuli used in the two patterning discriminations; generalization between the compounds would serve to reduce responding to the new compounds below that predicted by the Rescorla-Wagner model. When we compared responding to the two compounds during training, we found a significant difference, indicating that the rats did, at least to some extent, discriminate between the compounds. As a further investigation of this issue, after the test phase, we retrained half the rats (those run in the second replication) on a positive-patterning discrimination using one pair of stimuli (C and D) previously used in a negative-patterning schedule, while continuing negativepatterning training with the other pair of stimuli (A and B). The important observation was that, within a few days, the rats learned to respond more to the reinforced compound (CD) than to its nonreinforced single stimuli (C and D), while continuing to respond less to the $\mathrm{AB}$ compound than to its component stimuli. This again demonstrates that the rats could distinguish effectively between the compounds used in these discriminations.

\section{EXPERIMENT 3}

Experiment 3 used the same approach as that in Experiment 2 , but in a positive-patterning discrimination that inverted the reinforcement contingencies of the negativepatterning design. Rats were trained with intermixed trials of $\mathrm{A}-, \mathrm{B}-, \mathrm{AB}+, \mathrm{C}-, \mathrm{D}-$, and $\mathrm{CD}+$, before being tested with these stimulus configurations and the two novel configurations (AC and $\mathrm{BD}$ ). The role of a configural element in the positive-patterning discrimination has not been tested before. Such a test is important because any mechanism proposed to operate during a negative-patterning schedule should also operate during positive patterning. Thus, according to the added configural element hypothesis (Rescorla, 1972; Whitlow \& Wagner, 1972), the configural element should contribute to responding on $\mathrm{AB}+$ and $\mathrm{CD}+$ trials, and because this will not generalize to the $\mathrm{AC}$ and $\mathrm{BD}$ compounds, responding to those compounds should be lower. Nonetheless, responding to AC and BD should be above the level of responding elicited by the single CSs (unless rats achieve asymptotic discrimination performance, in which case responding to the single CSs and the new compounds will equal zero).

\section{Method}

\section{Subjects and Apparatus}

Sixteen experimentally naive female Hooded Wistar rats (weighing 190-253 g at the start of the experiment) were bred and maintained in the animal research colony in the School of Psychology at the University of Sydney. Three days prior to commencement of the experiment, their access to food was restricted to $2 \mathrm{~h}$ per day (from 3 to 5 p.m.). The rats were trained and tested in the same operant chambers and with the same stimuli as those in Experiment 1.

\section{Procedure}

Training. Before commencing training, the rats received context preexposure, stimulus preexposure, and magazine training across 3 consecutive days, as in Experiment 1. For the next 16 days, the rats were trained on two positive-patterning schedules. In each daily session, they received four 30 -sec presentations of each single stimulus and eight $30-\mathrm{sec}$ presentations of their two compounds ( $\mathrm{AB}$ and $\mathrm{CD}$ ). Presentation of the compounds, but not the single stimuli, terminated with the delivery of a food pellet. The stimuli were presented in a random order on a VT 2.5-min schedule, with the constraint that no more than two trials of the same type occurred consecutively. The number and duration of photobeam interruptions by head entry into the magazine was recorded during each $30-\mathrm{sec}$ stimulus and during the 30 -sec prestimulus period.

Test. The rats were tested once (Day 17) under extinction. They received four 30 -sec presentations of each of four cross-modal compounds ( $\mathrm{AB}, \mathrm{CD}, \mathrm{AC}$, and $\mathrm{BD})$, as well as two 30 -sec presentations of each of the four single stimuli. Trials were intermixed, and no food pellets were delivered.

\section{Results}

Two rats all but ceased responding after the 4th day of training (on average, they produced only one or two responses per stimulus) and were therefore removed from all further analysis. The other 14 rats showed consistently higher response rates to each compound than to both of the constituent stimuli across the last 4 days of training. As can be seen in Figure 3, these rats increased their response rate to the reinforced compounds across the 16 days of training, reaching, on average, about 14 responses per stimulus presentation. In contrast, responding to the nonreinforced single stimuli remained constant at about 7 responses per presentation. The difference in responding between compound and single stimulus trials was significant on each of the last 5 days of training [smallest $t(13)=$ 4.72, all $p \mathrm{~s}<.01]$.

When tested under extinction, the average number of responses made during presentations of the single stimuli (A, B, C, and D) was less than that to the trained compounds (AB and $\mathrm{CD}$ ) and the new compounds (AC and BD). An ANOVA revealed a significant overall effect $[F(2,26)=73.51, p<.01]$. Contrasts comparing responding to the new compounds with that to the trained CSs and compounds established that the rats responded significantly more to the new compounds than to the single stimuli $[F(1,13)=67.65, p<.01]$, but the difference in 


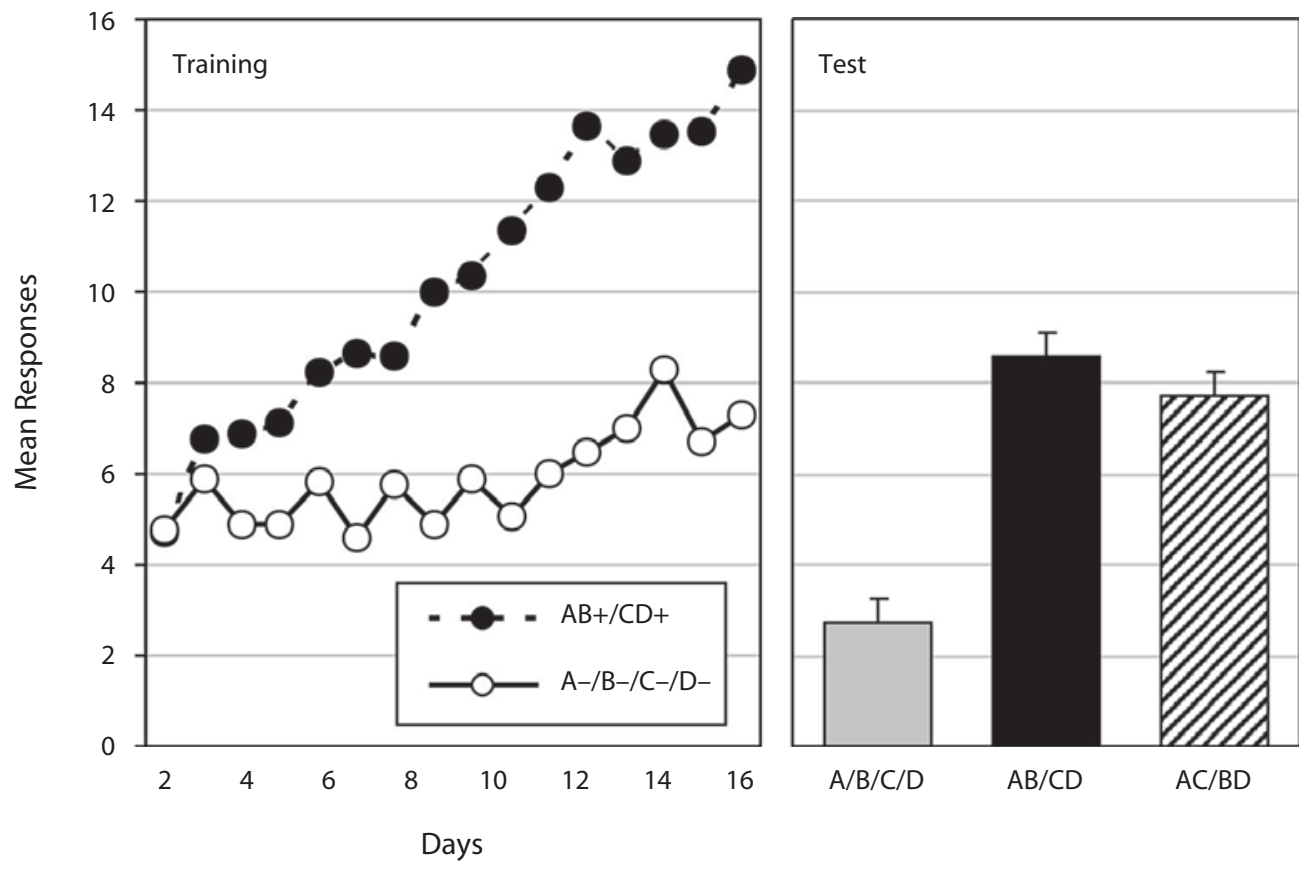

Figure 3. Left: Mean number of responses (magazine entries) above baseline during reinforced presentations of compound stimuli (AB and $C$ ) and nonreinforced presentations of single stimuli $(A, B, C$, and $D)$ during positive-patterning training in Experiment 3. Right: Mean number of responses to the same single and compound stimuli, as well as two new compounds (AC and BD) composed of novel combinations of the trained stimuli, on subsequent test. Only data from the 13 rats that successfully acquired both patterning discriminations are plotted here.

responding to the new and old compounds fell short of conventional significance $[F(1,13)=3.96, p=.068]$.

\section{Discussion}

Rats trained in two concurrent positive-patterning discriminations learned to respond more to the reinforced stimulus compounds ( $\mathrm{AB}$ and $\mathrm{CD})$ than to their nonreinforced component stimuli (A, B, C, and D). Subsequent presentation of novel combinations of those stimuli (AC and $\mathrm{BD}$ ) also elicited high levels of responding that were slightly, but not significantly, less than the level elicited by the trained compounds. Because of this difference, the present results are inconclusive with regard to the Rescorla-Wagner model, which predicts that responding to the new compounds will be below that to the trained compounds (Rescorla, 1972; Whitlow \& Wagner, 1972).

\section{EXPERIMENT 4}

Experiment 4 combined the discriminations used in Experiments 2 and 3 , so that rats were trained simultaneously on a negative- and a positive-patterning discrimination (i.e., they received intermixed trials of $\mathrm{A}+, \mathrm{B}+, \mathrm{AB}-$, $\mathrm{C}-, \mathrm{D}-$, and $\mathrm{CD}+$ ), before being tested with these single or compound stimuli and the two novel compounds (AC and $\mathrm{BD}$ ). Thus, as in Experiments 2 and 3, this design tested responding to novel compounds composed of stimuli with mixed reinforcement histories. But unlike in those experiments, it removed the correlation between stimulus number (single vs. compound) and reinforcement. Therefore, as in Experiment 1, this experiment removed the possibility that the rats might learn during training to respond to all the compounds or withhold responding to all the compounds, which would clearly affect their response to the new compounds. Our initial attempts to train rats on both discriminations simultaneously revealed the difficulty of the task, and therefore, in this experiment, the number of trials per day was increased substantially to 64 trials per day for 50 days. Data from the training phase of this experiment have been reported in a separate publication that compares the rate at which rats learn these discriminations with the rate at which rats learn a biconditional discrimination (Harris, Livesey, Gharaei, \& Westbrook, 2008). Here, we report the data from two posttraining tests that involved nonreinforced presentations of the novel compounds ( $\mathrm{AC}$ and $\mathrm{BD}$ ), intermixed among trials from the two trained patterning discriminations. According to the added configural element hypothesis (Rescorla, 1972; Whitlow \& Wagner, 1972), responding to the novel compounds should be given by the summed associative strength of the individual component stimuli. Therefore, depending on the level of responding to the nonreinforced single $\mathrm{CSs}(\mathrm{C}$ and $\mathrm{D})$, responding to $\mathrm{AC}$ and $\mathrm{BD}$ should be equal to or greater than responding to CSs A and B.

\section{Method}

\section{Subjects, Apparatus, and Procedure}

Sixteen experimentally naive male Hooded Wistar rats were trained and tested in the same operant chambers and with the same 

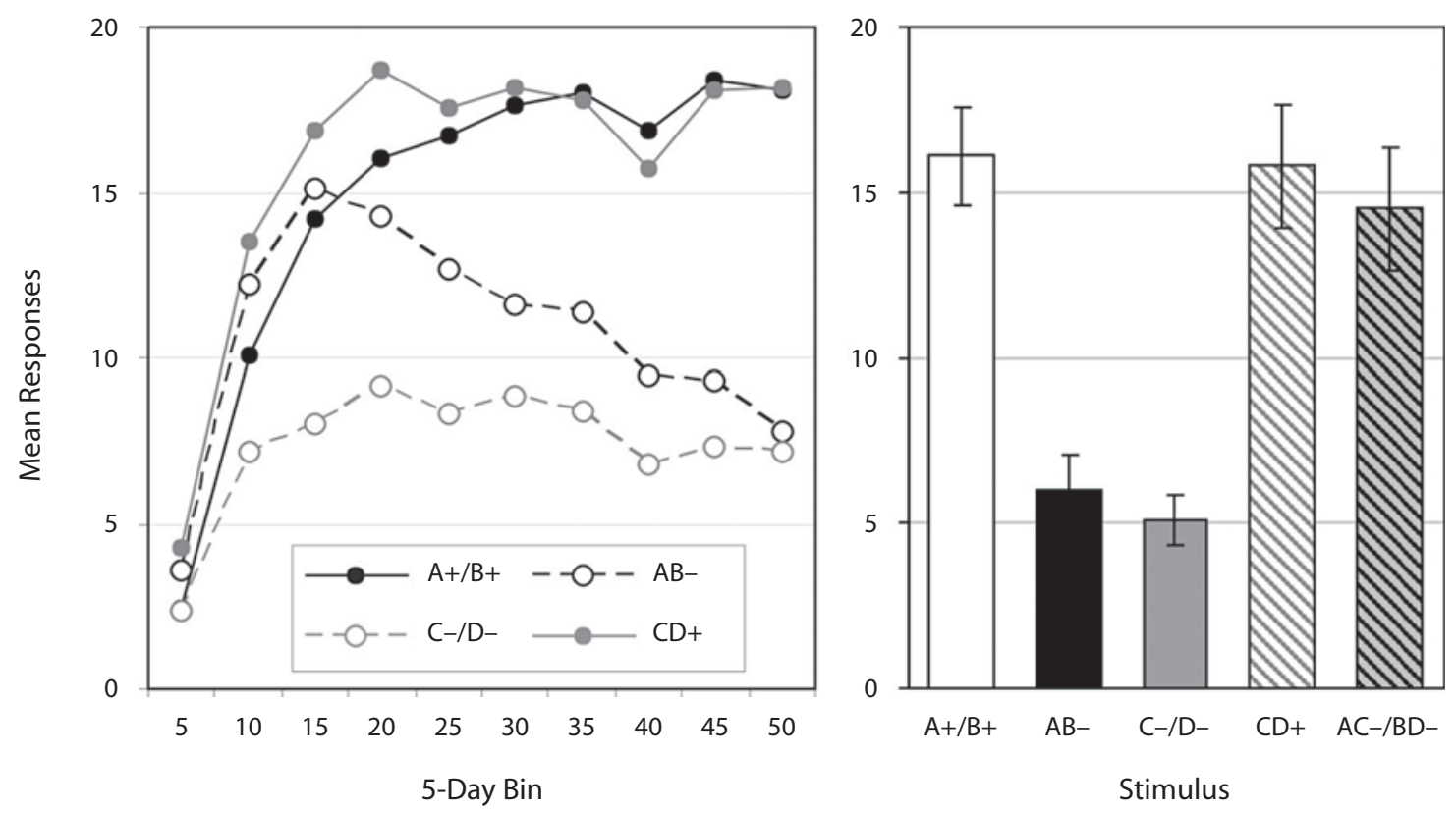

Figure 4. Mean number of responses (magazine entries) above baseline during training (left) and test (right) presentations of single and compound conditioned stimuli in Experiment 4 . The rats had been trained concurrently on a negativepatterning discrimination $(A+B+v s$. $A B-)$, as well as a positive-patterning discrimination $(C-D-v s$. $C D+)$. $A$ detailed analysis of the training data from this experiment is presented in Harris, Livesey, Gharaei, and Westbrook (2008).

stimuli as those in the previous experiments. Details for the rats and the training procedures have been reported in Harris et al. (2008). In brief, the rats were trained for 50 days, with 64 trials per day. These comprised $16 \mathrm{CD}+$ trials, $16 \mathrm{AB}-$ trials, $8 \mathrm{C}-$ and $8 \mathrm{D}-$ trials, and $8 \mathrm{~A}+$ and $8 \mathrm{~B}+$ trials, randomly intermixed. All stimulus presentations were of 30 -sec duration and were on the VT 2.5-min schedule used in Experiments 1-3. On Days 51 and 52, the rats were tested. These 2 test days consisted of 24 negative-patterning trials $(6 \mathrm{~A}+$, $6 \mathrm{~B}+$, and $12 \mathrm{AB}-$ trials $), 24$ positive-patterning trials $(6 \mathrm{C}-$, $6 \mathrm{D}-$, and $12 \mathrm{CD}+$ trials), and 8 nonreinforced trials with the two novel compounds (4 $\mathrm{AC}-$ and $4 \mathrm{BD}-$ trials). All trial types were randomly intermixed, with the exception that the new compounds were not presented in the first third of the test session, so that the rats received two full cycles of both discriminations before the novel compounds were introduced. As a result, only the data from the last two thirds of the test session have been included in the analyses described below.

\section{Results}

All the rats reached the criterion used in Experiments $1-3$, in that they responded more on reinforced than on nonreinforced trials on at least 4 of the last 5 days, for both discriminations. Although the rats took longer to learn the negative-patterning discrimination (Harris et al., 2008), their terminal performance on both discriminations was equivalent, with the mean number of responses on reinforced and nonreinforced trials differing by 10 responses per 30-sec stimulus for both positive and negative patterning. An ANOVA on the last 5 days of training confirmed that there was a significant overall difference between reinforced and nonreinforced trials $[F(1,15)=$ $247.65, p<.01]$ but no overall difference between the positive- and negative-patterning discriminations $(F<1)$ and no interaction between discrimination and reinforce- ment $(F<1)$. A summary of the training data is shown on the left side of Figure 4.

The mean number of responses to each single or compound CS during the last two thirds of both test sessions is shown on the right side of Figure 4. An ANOVA revealed a significant overall effect on test $[F(1,15)=32.32, p<$ $.01]$. The rats continued to discriminate well between reinforced and nonreinforced trials on both discriminations, responding more on $\mathrm{A}+$ and $\mathrm{B}+$ trials than on $\mathrm{AB}-$ trials and also more on $\mathrm{CD}+$ trials than on $\mathrm{C}-$ and $\mathrm{D}-$ trials. Of particular interest are the response rates to the novel compounds (AC and BD). As is clear in Figure 4, the rats responded more to these compounds than to $\mathrm{AB}-$ or to $\mathrm{C}-$ and $\mathrm{D}-$. A statistical analysis confirmed that these differences were significant $[F(1,15)=22.24$ and $46.18, p \mathrm{~s}<.01]$. However, responses to $\mathrm{AC}$ and $\mathrm{BD}$ did not differ significantly from response rates to $\mathrm{A}+$ and $\mathrm{B}+$ $[F(1,15)=1.79, p=.200]$ or to $\mathrm{CD}+[F(1,15)=2.14$, $p=.164]$.

\section{Discussion}

Rats trained on concurrent negative- and positivepatterning discriminations learned to respond more on reinforced trials $(\mathrm{A}+, \mathrm{B}+$, and $\mathrm{CD}+)$ than on nonreinforced trials $(\mathrm{AB}-, \mathrm{C}-$, and $\mathrm{D}-)$. Test presentations of novel combinations of those stimuli (AC and BD) also elicited high levels of responding that were slightly but not significantly below the levels of responding on reinforced trials. The Rescorla-Wagner model with an added configural element (Rescorla, 1972; Whitlow \& Wagner, 1972) predicts greater responding to $\mathrm{AC}$ and $\mathrm{BD}$ than to $\mathrm{A}$ and $\mathrm{B}$, but only if $\mathrm{C}$ and $\mathrm{D}$ retain enough associa- 


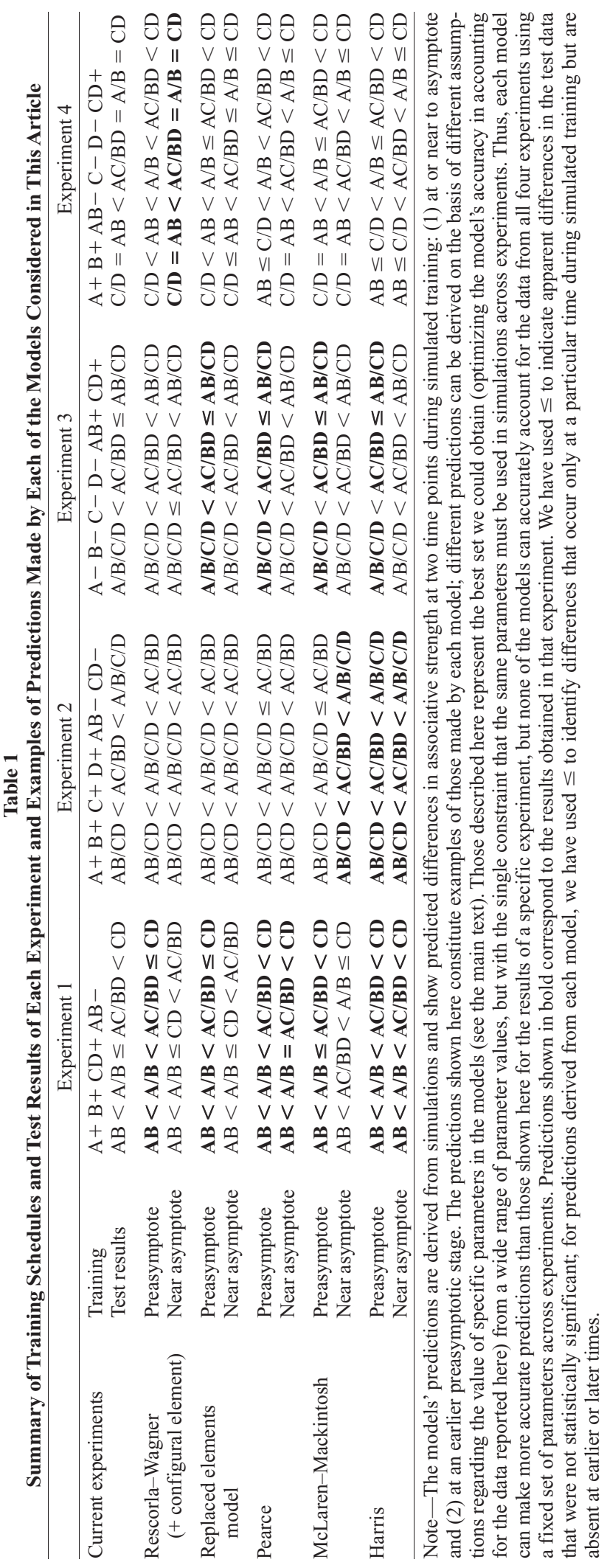


tive strength to summate with the associative strength of A and B. However, the Rescorla-Wagner model can predict the results obtained here if performance on the patterning discriminations were close to asymptote, so that $\mathrm{C}$ and D effectively had no associative strength. Evidence for this is equivocal. On the one hand, responding to $\mathrm{C}$ and $\mathrm{D}$ (and $\mathrm{AB}$ ) was above baseline, suggesting that the rats had not yet reached asymptotic performance. On the other hand, the fact that discrimination performance on the negative-patterning discrimination had reached the same level as performance on the positive-patterning discrimination suggests that performance on these discriminations was effectively at asymptote. As such, the findings of Experiment 4 would support the predictions of the added configural elements hypothesis as incorporated within the Rescorla-Wagner model.

\section{GENERAL DISCUSSION}

The four experiments presented here investigated rats' responses to single and compound stimuli after training on patterning discriminations. In Experiment 1, rats were trained on a negative-patterning schedule $(\mathrm{A}+\mathrm{B}+\mathrm{AB}-)$ intermixed with reinforced presentations of a second compound $(\mathrm{CD}+)$. Once trained, the rats were tested with two new stimulus compounds composed of novel combinations of the trained stimuli (AC and $\mathrm{BD}$ ). The rats responded more to the new compounds than to the individual CSs (A, B, C, and D) or the $\mathrm{AB}$ compound, but responding to the new compounds was below that to the $\mathrm{CD}$ compound. In Experiments 2 and 3, rats were trained in two concurrent patterning discriminations: two negative-patterning discriminations in Experiment $2(\mathrm{~A}+\mathrm{B}+\mathrm{AB}-, \mathrm{C}+\mathrm{D}+$ $\mathrm{CD}-)$ and two positive-patterning discriminations in Experiment $3(\mathrm{~A}-\mathrm{B}-\mathrm{AB}+, \mathrm{C}-\mathrm{D}-\mathrm{CD}+)$. Again, the rats were tested with the new compounds ( $\mathrm{AC}$ and $\mathrm{BD}$ ). In Experiment 2, they responded more to the new compounds than to the trained compounds, but less than to the individual CSs. In Experiment 3, they responded as much to the new compounds as to the trained compounds and more than to the individual CSs. Finally, in Experiment 4, rats that had been trained on concurrent negative- and positive-patterning discriminations $(\mathrm{A}+\mathrm{B}+\mathrm{AB}-, \mathrm{C}-$ $\mathrm{D}-\mathrm{CD}+$ ) were tested with the new compounds ( $\mathrm{AC}$ and $\mathrm{BD}$ ). Levels of responding to the new compounds were very close to the response rates observed on reinforced trials of the patterning discriminations. Below, we will discuss the implications of these findings for theories of stimulus representation in associative learning. We first will consider the conventional account in which configural elements that uniquely represent each compound are added to the representation of the component stimuli (Rescorla, 1972; Spence, 1952; Whitlow \& Wagner, 1972). We will also consider the implications of these data for a number of more recent models of stimulus representation (Harris, 2006; McLaren \& Mackintosh, 2002; Pearce, 1987, 1994; Wagner \& Brandon, 2001). Table 1 summarizes the training schedule and test results for each experiment, as well as the predictions made by each of the models for each experiment. These predictions are derived from simulations of the associative strength for each of the training and test stimuli, calculated on a trial-by-trial basis for an extended course of "training." Table 1 presents the predicted differences in associative strength at two stages of simulated training: at or near asymptotic performance and at an earlier stage of performance (where the relative ordering of the stimuli differ from those at asymptote). We have done this in order to account for the fact that, in the experiments presented here, we cannot be sure how close the animals were to asymptotic performance on each discrimination at the time of testing.

\section{The Added Configural Element Hypothesis}

As was described in the introduction, there is a long tradition that considers stimuli to be represented by discrete elemental units, each of which enters into association with the US (Atkinson \& Estes, 1963; Bush \& Mosteller, 1951; Estes, 1950). To explain how animals can master patterning discriminations - and in particular, negative patterning - it has been suggested that compounds of two or more stimuli are represented by the individual elements of the component stimuli plus a configural element that uniquely codes that combination of stimuli (Rescorla, 1972; Spence, 1952; Whitlow \& Wagner, 1972). The results from Experiments 1, 2, and 4 offer some support for this view, since it predicts, correctly, that the rats should respond more to the new compounds than to the negativepatterning compounds, because responding to the latter (but not the former) compounds would be reduced by the inhibitory strength acquired by their configural elements. Moreover, if the rats' performance on the two patterning discriminations had reached asymptote in Experiment 4, the Rescorla-Wagner model with an added configural element would correctly predict that responding to the novel compounds $\mathrm{AC}$ and $\mathrm{BD}$ would equal responding to the reinforced CSs (A and B) and the CD compound. However, this account also predicts, incorrectly, that the net associative strength of the new compounds would be greater than that of every other stimulus configuration tested in Experiments 1 and 2. That is, the rats should have responded more to $\mathrm{AC}$ and $\mathrm{BD}$ than to $\mathrm{CD}$ in Experiment 1 and should have responded more than to the individual CSs (A, B, C, and D) in Experiment 2. Neither of these predictions was confirmed. Furthermore, with regard to Experiment 3, the Rescorla-Wagner model predicts that responding to the new compounds should be less than that to the trained compounds, because any associative strength acquired by the configural elements of the trained compounds would not generalize to the new compounds. Responding to the new compounds in Experiment 3 was below that to the trained compounds, but the difference was small and was not statistically significant. Finally, the Rescorla-Wagner model predicts that, at asymptote, responding to the single CSs A and B in Experiment 1 should equal that to the $\mathrm{CD}$ compound, whereas in that experiment, responding to $\mathrm{A}$ and $\mathrm{B}$ remained well below that to $\mathrm{CD}$.

The present difficulties for the Rescorla-Wagner model stem from two particular assumptions: (1) that the associative strength of a compound is equal to the summed strengths of the individual CSs and (2) that the configural 
elements of each compound are unique. The first assumption forces the model to predict much higher levels of responding to the new compounds than to their component CSs (see Thein, Westbrook, \& Harris, 2008, for an investigation of summation of responding in this paradigm). This is particularly problematic in Experiment 2, in which responding to the new compounds was below that to the single CSs. Thus, the model's predictions would be improved if it incorporated a description of the representation of stimuli that would anticipate some form of generalization decrement in associative strength between single CSs and their compounds. This issue will be returned to below.

The second assumption, about the uniqueness of configural elements, is problematic because it does not anticipate generalization between compounds other than via their shared CS elements. Thus, once again, the model could provide more accurate predictions about the present results if it were to assume that some of the associative strength acquired by the configural element of any compound could generalize to other compounds. Specifically, in Experiment 2, if the inhibitory associative strength acquired by the configural elements of the trained compounds were to generalize to the new compounds, this would reduce responding to those compounds. Similarly, if excitatory associative strength acquired by the configural elements of the trained compounds in Experiment 3 were to generalize to the new compounds, this would increase responding to those compounds. Such arguments are less applicable to the results of Experiments 1 and 4, because generalization of inhibition from the configural element of $\mathrm{AB}$ would be offset by generalization of excitation from the configural element of CD. Nonetheless, in both of those experiments, generalized excitation from CD would not completely cancel generalized inhibition from $\mathrm{AB}$, because, according to the Rescorla-Wagner model, AB's inhibitory strength should be greater than CD's excitatory strength. This would serve to reduce the predicted level of responding to the novel compounds, relative to the single reinforced CSs, in Experiments 1 and 4. It is also worth pointing out that although Experiment 2 provided evidence that the rats did discriminate effectively between compounds, this does not exclude the possibility of partial generalization between compound configural elements.

Before finishing our discussion of the Rescorla-Wagner model, it is worth noting that patterning discriminations can be solved by that model without resort to a unique configural element. To achieve this, the model must assume that the context (or some equivalently ubiquitous element) functions as a salient but redundant cue, so that negative patterning takes the form $\mathrm{AX}+\mathrm{BX}+\mathrm{ABX}-$ and positive patterning becomes $\mathrm{AX}-\mathrm{BX}-\mathrm{ABX}+$. This approach can solve these discriminations because, according to the Rescorla-Wagner model, the redundant cue $\mathrm{X}$ acquires excitatory strength during negative patterning and acquires inhibitory strength during positive patterning (Rescorla, 1972). This account predicts that responding to the new compounds should be equivalent to that to the trained compounds and, thus, can correctly account for the results of Experiment 3, but not for those of Experiments 1 and 2. Moreover, because the redundant cue $\mathrm{X}$ must acquire excitatory strength during negative patterning but inhibitory strength during positive patterning, this account cannot be invoked as a solution to the concurrent positive- and negative-patterning discriminations that were mastered by the rats in Experiment 4.

\section{The Replaced Elements Model}

Wagner and Brandon (2001) have recently revised the added configural element approach in describing the representations of single and compound stimuli (see also Wagner, 2003). In their model, when stimuli are combined as a compound, configural elements that are unique to the compound replace some elements of the individual stimuli (this differs from the Rescorla-Wagner model, in which configural elements are simply added to the full array of elements). Because some elements of the individual stimuli are lost (replaced) in the compound, some of the associative strength of individual CSs does not generalize to their compound. By parameterizing the proportion of elements that can be replaced, the model can anticipate a greater or lesser associative decrement when single stimuli are combined as a compound. In this way, the replaced elements model can explain why responding to the novel compounds in Experiments 1 and 2 was lower than is predicted by the Rescorla-Wagner model. However, our simulations of this model reveal that it cannot simultaneously explain the findings from all four experiments present here if it is constrained, as it should be, to assuming a constant level of replacement across all experiments. In particular, it must assume that a high proportion $(>50 \%)$ of elements are replaced in order to account for the fact that responding to the novel compounds in Experiment 2 was below responding to the single CSs, yet it must also assume that a low proportion $(<5 \%)$ of elements are replaced to account for the high level of responding to the novel compounds in Experiments 3 and 4. Furthermore, like the Rescorla-Wagner model, the replaced elements model anticipates that asymptotic responding to $\mathrm{A}$ and $\mathrm{B}$ should equal that to $\mathrm{CD}$ in Experiment 1, in contrast to the observation that responding to $\mathrm{A}$ and $\mathrm{B}$ reached a plateau well below that to CD in that experiment.

In investigating the predictions of this model, we have also considered how similarity between stimuli from the same modality will affect generalization between single and compound stimuli. Specifically, if we assume that Stimulus B and Stimulus C share some proportion of their elements in common because they belong to the same sensory modality (and likewise, that A and D share elements in common), these common elements will support generalization both between the single stimuli and between their compounds. But more crucially, these common elements may support generalization of associative strength between configural elements of different compounds. For example, when $\mathrm{A}$ is compounded with $\mathrm{B}$, the $\mathrm{B}$ elements that are replaced by configural elements will include some that are common between B and C. It is reasonable to assume that exactly the same common elements will be replaced by exactly the same configural elements when $\mathrm{A}$ is compounded with $\mathrm{C}$. We assume this because, in each case, $\mathrm{A}$ is compounded with the same set of $\mathrm{B} / \mathrm{C}$ 
elements. Therefore, some configural elements in the $\mathrm{AB}$ compound will not be unique to that compound but will also be activated by the $\mathrm{AC}$ compound, supporting further generalization between $\mathrm{AB}$ and $\mathrm{AC}$ (but not between their individual CSs). Our simulations of this operation reveal that increasing the number of common elements between stimuli from the same modality decreases the associative strength of the novel AC and BD compounds in Experiments 1, 2, and 4 but increases it in Experiment 3. This improves the ability of the replaced elements model to predict the findings from all the experiments presented here, but our exploration of the parameter space (varying both proportion of replacement and similarity) did not identify a combination of parameters that would successfully reproduce the outcomes of all four experiments. In particular, to explain the level of responding to the novel compounds in Experiment 4, it was necessary to assume levels of replacement and similarity that were too low to account for the level of responding to those compounds in Experiments 1 and 2.

\section{Pearce's Configural Model}

In a notable departure from the traditional elemental view of stimulus representation, Pearce $(1987,1994$, 2002) rejected the notion that associative processes act at the level of stimulus elements and, instead, argued that associative strength accrues to single configural units that represent entire patterns of stimulus input. Thus, presentation of an individual stimulus (A) activates a configural unit $(A)$ that is distinct from the configural unit $(A B)$ activated by presentation of the $\mathrm{AB}$ compound (we use italics to denote the configural unit for each stimulus pattern). In addition to activating its corresponding configural unit, a given pattern of stimulus input also partially activates other configural units, and the strength of this generalized activation is given by a product rule incorporating the proportion of overlap between that input pattern and the pattern coded by the configural unit in question.

Patterning discriminations are solved in Pearce's model because the reinforced and nonreinforced stimulus patterns correspond to different configural units: In negative patterning, excitatory associative strength is confined to the $A$ and $B$ units, whereas $A B$ acquires inhibitory strength; in positive patterning, excitatory strength is confined to the $A B$ unit, whereas $A$ and $B$ acquire inhibitory strength. With regard to the present experiments, the new $\mathrm{AC}$ and $\mathrm{BD}$ compounds would elicit responding proportional to the degree to which they activate the configural units of each of the trained stimulus patterns. For example, AC would activate the $A$ and $C$ units to half strength each and would activate the $A B$ and $C D$ units to one-fourth strength. Therefore, responding to $\mathrm{AC}$ will be given by $1 / 2 V_{A}+$ $1 / 2 V_{C}+1 / 4 V_{A B}+1 / 4 V_{C D}$ (where $V_{i}$ is the associative strength of unit $i$ ). When we compare this with the associative strength activated by $\mathrm{A}\left(=V_{A}+1 / 2 V_{A B}\right)$ and $\mathrm{C}\left(=V_{C}+\right.$ $\left.1 / 2 V_{C D}\right)$ individually, we can see that this account predicts that responding to the new compounds will equal the average level of responding to the individual stimuli [e.g., $\mathrm{AC}=(\mathrm{A}+\mathrm{C}) / 2]$. Thus, the model predicts less respond- ing than was observed in Experiments 1, 3, and 4 but more responding than was observed in Experiment 2.

The description of the Pearce model above takes a simplified position in which stimulus presentations are viewed as completely distinct and isolated events. Pearce (1994) pointed out that the details of the model are somewhat different if one takes into consideration the fact that stimuli are likely to bear some similarity to one another and that the training context is likely to be a significant part of the stimulus patterns encoded by each configural unit. These considerations will increase generalized activation of configural units by different input patterns. The specific details of the model's predictions can also change if the experimental context is included as part of each configural representation.

To discover what specific predictions the model makes for the patterning experiments presented here, we have simulated the net associative strength retrieved by different stimulus inputs. In these simulations, we varied the similarity between stimuli from the same modality (i.e., between $\mathrm{A}$ and $\mathrm{D}$, and between $\mathrm{B}$ and C), as well as varying the salience of the context, relative to that of the other stimuli. These simulations revealed the following rules. Increasing similarity between stimuli from the same modality increases generalization between the trained and new compounds and, therefore, reduces the predicted response to $\mathrm{AC}$ and $\mathrm{BD}$ following negative patterning but increases it following positive patterning. Thus, generalization between stimuli from the same modality can enable Pearce's model to make accurate predictions for Experiments 2 and 3 but does not improve its predictions for Experiment 1 or 4. On the other hand, increasing the salience of the context in the configural representations of each trial increases summation of responding to compounds and, therefore, increases predicted responding to $\mathrm{AC}$ and $\mathrm{BD}$ following either negative or positive patterning. Thus, generalization based on salient contextual elements improves the model's predictions for Experiments 1,3 , and 4 , but its predictions become less accurate for Experiment 2.

To test Pearce's model against the present data, we conducted many simulations searching the parameter space to identify a single set of parameter values that would enable the model to account for all the experiments simultaneously. Our search failed to find such a set of parameters. For Experiment 4, a high value for context salience (five times greater than each stimulus) and little or no similarity between stimuli is required for the model to predict that the associative strength of $\mathrm{AC}$ and $\mathrm{BD}$ will be close to that of A, B, and CD. However, these same values for context salience and similarity produce estimations of the associative strength of $\mathrm{AC}$ and $\mathrm{BD}$ in Experiment 2 that are greater than the associative strength of each single CS. For Experiment 2, context salience must be low and/ or similarity between stimuli must be high for the model to generate an estimate of the associative strength of $\mathrm{AC}$ and BD that is below that of the single CSs. Finally, it is worth noting that inclusion of the context as part of each configural representation leads Pearce's model to predict 
that asymptotic responding to $\mathrm{A}$ and $\mathrm{B}$ will be below that to $\mathrm{CD}$ in Experiment 1, as was observed.

\section{The McLaren-Mackintosh and Harris Models}

Two relatively recent models have provided alternative solutions to negative patterning that do not require the assumption that compounds are distinguished from their component stimuli by configural elements. In both models, stimuli are represented by an array of elemental units that correspond to distinct sensory features of the stimulus, and each element individually acquires excitatory or inhibitory associative strength across reinforced and nonreinforced trials according to the simple delta rule described in the Rescorla-Wagner model. The elements representing a compound are the same as those representing the individual stimuli, but the activation strength of many elements will differ between the compound and the individual stimuli (or, if the element is common to both stimuli, its activation in the compound will differ from the simple sum of its activation in the single stimuli). Thus, this quantitative, rather than qualitative, difference in the representation of single and compound stimuli enables both models to solve negative-patterning discriminations. We will provide more specific details of both models below and will discuss the implications of the present findings.

In the McLaren-Mackintosh (2002) model, the representation of any single stimulus is distributed across half the total representational space, and as a result, any two stimuli can be expected to share $50 \%$ of their elements in common. The relationship between the intensity of a particular feature in the stimulus and the activation strength of the corresponding element follows a steep sigmoid function, so that activation of elements is effectively binary: Elements are either on or off. This means that, when two stimuli are presented as a compound, the large proportion of elements that they share in common will be activated in the same way (to the same strength) in the compound as in each individual stimulus. The model can solve a negative-patterning discrimination because, as has been pointed out by Rescorla (1972), the common elements acquire excitatory associative strength, whereas the distinctive elements of each stimulus acquire inhibitory associative strength.

The McLaren-Mackintosh (2002) model can account for the response rates to the novel compounds, relative to the trained single and compound stimuli, in Experiments 1, 2 , and 3 if one makes the reasonable assumption that the number of common elements between stimuli from the same modality is greater than that between stimuli from different modalities. Unfortunately, this assumption does not help the model to account for the finding in Experiment 4 that responding to the novel compounds was close to that to the reinforced single CSs and the reinforced compound. Rather, the model consistently predicts that the associative strength of the novel compounds should be midway between that of the two trained compounds. The model's predictions for Experiment 4 can be improved by relaxing the rule that all stimuli should share at least $50 \%$ of their elements in common. If stimuli share a much smaller proportion of common elements (implying that the stimuli used here engage much less than half the total representational space), the model can correctly predict that the associative strength of the novel compounds will be close to that of the reinforced CSs and compound. However, this also changes the model's predictions for the other experiments and, in particular, forces the model to predict incorrectly that the associative strength of the novel compounds in Experiment 2 will be greater than that of the single CSs. Our search of the parameter space, varying the amount of overlap between stimuli from the same modality and from different modalities, did not identify a set of parameters that enabled this formulation of the model to reproduce accurately the pattern of results from all four experiments. Moreover, our simulations of the model indicated that, like the Rescorla-Wagner and replaced elements models, it consistently predicts that asymptotic responding to $\mathrm{A}$ and $\mathrm{B}$ should equal that to $\mathrm{CD}$ in Experiment 1. Thus, the model does not appear to be able to anticipate the observation in that experiment that asymptotic response rates to $\mathrm{A}$ and $\mathrm{B}$ remained well below those to CD.

The analysis of the McLaren-Mackintosh model above treats element activation as binary; elements are either active $(=1)$ or inactive $(=0)$, so that all the elements active in the compound are active in at least one of the individual stimuli, whereas all the elements active in either single stimulus are active in the compound. However, the authors of that model have suggested that this oneto-one mapping may not hold if elements have what is effectively subthreshold activation or even negative activation (McLaren \& Mackintosh, 2002). Any element that receives subthreshold or negative activation in a stimulus is effectively inactive in that stimulus, but this type of activation may have different consequences for its activation in a compound. First, if an element has subthreshold activation in both of two individual stimuli, it may receive suprathreshold activation in the compound of those stimuli, because the combination of the two subthreshold inputs may exceed the activation threshold. Thus, an element may be fully activated $(=1)$ in the compound but effectively inactive $(=0)$ in each individual stimulus (much like an added configural element in the Rescorla-Wagner model). Second, an element that receives negative activation in one stimulus may remain inactive in the compound even if it receives suprathreshold activation by the other stimulus of the compound. That is, an element may be active in one of the individual stimuli but suppressed in the compound. We have incorporated these operations in further simulations of the model. In general, such "added" or "suppressed" compound elements facilitate learning of the patterning discriminations, and, by reducing generalization between individual CSs and their compound, these elements reduce the predicted associative strength retrieved by the novel compounds in Experiments 1 and 2. However, these improvements in the model's predictions are offset by the fact that these same elements reduce the predicted associative strength of the novel compounds in Experiment 4. Once again, our search of the parameter space was unable to identify any combination of the 
four parameters (overlap between and within modality, proportion of added compound elements, and proportion of suppressed compound elements) that satisfactorily reproduced the basic pattern of findings from all four experiments simultaneously. However, we note that we have offered only a trial-based analysis of the McLarenMackintosh model here. A real-time implementation of the model may produce different predictions with regard to the present experimental designs.

The elemental model proposed by Harris (2006) uses a capacity-limited attention buffer to introduce nonlinearity in the activation of stimulus elements. Elements compete for entry to the buffer, which boosts their activation strength multiplicatively. Elements that are strongly activated by a stimulus will compete successfully for entry to the buffer when the stimulus is presented in isolation or in compound, whereas more weakly activated elements gain entry to the buffer during presentation of the stimulus in isolation but are displaced from the buffer when that stimulus is presented in a compound. Because activation weight determines associability ( $\alpha$ in the Rescorla-Wagner model) and influences the expression of associative strength, the attention buffer affects both learning and performance. The attention buffer also determines the direction of change of associative strength: Associative strength between CS and US elements increases when the US elements are activated in the buffer, but associative strength decreases when the US elements are active outside the buffer. These basic operations of the model allow it to solve negative-patterning discriminations via two processes. First, because the weaker CS elements have lower activation strength during nonreinforced compound trials than during reinforced single CS trials, these elements can acquire most of the excitatory associative strength, and this associative strength will be expressed more strongly during the single CS than during the compound. Second, on each compound trial, the stronger elements of each stimulus will be active inside the attention buffer, whereas the weaker elements will be active outside the buffer. This will lead to inhibitory associations forming between the strong elements of each stimulus and the weaker elements of the other stimulus (e.g., the stronger elements of A will come to inhibit the weaker elements of B). This will further suppress expression of the excitatory associative strength of the weaker elements and, thereby, reduce responding during presentation of the compound.

The two processes described above enable the Harris (2006) model to account for the observed response rates to the novel compounds in the experiments presented here, but once again this cannot be achieved across all four experiments using a single set of parameters (the parameters we have explored describe similarity between stimuli from the same modality or different modalities, the learning rate for CS-US associations, and the learning rate for CS-CS associations). Parameters that enable the model to account for the high response rate to the novel compounds in Experiment 4 also cause the model to overestimate responding to those compounds in Experiment 2 and to underestimate it in Experiment 3, whereas parameters that produce appropriate estimates of responding to the novel compounds in Experiments 2 and 3 lead the model to underestimate responding to those compounds in Experiment 4. Nonetheless, like Pearce's $(1987,1994)$ configural model, but unlike the other three models considered here, Harris's model can successfully account for the observation in Experiment 1 that asymptotic responding to $\mathrm{A}$ and $\mathrm{B}$ was well below that to $\mathrm{CD}$, but only if the model assumes overlap in the representations of stimuli.

\section{Conclusions}

The four experiments reported in this article provide qualified support for different theoretical accounts of stimulus representation. Only the final experiment is consistent with the predictions of the Rescorla-Wagner model incorporating added configural elements (Rescorla, 1972; Whitlow \& Wagner, 1972). The results of Experiments $1-3$ contradict the predictions of that model. In contrast, the results of the first three experiments can be explained by more recent models of stimulus representation that involve purely elemental representations (Harris, 2006; McLaren \& Mackintosh, 2002), purely configural representations (Pearce, 1987, 1994), or a combination of elemental and configural representations (Wagner, 2003; Wagner \& Brandon, 2001). However, although each of these models can also account for the results of Experiment 4, the assumptions they must make to do so render them less able to explain the results of the earlier experiments. None of the models considered here can explain all four experiments if they are constrained to make consistent assumptions across experiments about the details underlying the representations of stimuli. This conclusion calls into question a key assumption in each approach, that the solution to patterning discriminations derives from fixed processes underlying stimulus representation. Thus, the present experiments may highlight the need for descriptions of stimulus representation to incorporate mechanisms that are sensitive to the output of the associative-learning process. An appropriate starting point would be to consider how the salience of elemental or configural units may change as a consequence of their associative strength, using rules that have been formalized in existing models of associative learning (e.g., Le Pelley, 2004; Mackintosh, 1975; Pearce \& Hall, 1980).

\section{AUTHOR NOTE}

This work was supported by Discovery Grant DP0451764 from the Australian Research Council. The authors thank Bob Boakes, Fred Westbrook, and Evan Livesey for helpful comments. Correspondence concerning this article should be addressed to J. A. Harris, School of Psychology, University of Sydney, Sydney 2006, NSW, Australia (e-mail: justinh@psych.usyd.edu.au).

\section{REFERENCES}

Atkinson, R. C., \& Estes, W. K. (1963). Stimulus sampling theory. In R. D. Luce, R. B. Bush, \& E. Galanter (Eds.), Handbook of mathematical psychology (Vol. 3, pp. 121-268). New York: Wiley.

Bush, R. R., \& Mosteller, F. (1951). A model for stimulus generalization and discrimination. Psychological Review, 58, 413-423. doi: $10.1037 / \mathrm{h} 0054576$ 
Estes, W. K. (1950). Towards a statistical theory of learning. Psychological Review, 57, 94-107. doi:10.1037/h0058559

HARris, J. A. (2006). Elemental representations of stimuli in associative learning. Psychological Review, 113, 584-605. doi:10.1037/0033 $-295 X .113 .3 .584$

Harris, J. A., Livesey, E. J., Gharaei, S., \& Westbrook, R. F. (2008). Negative patterning is easier than a biconditional discrimination. Journal of Experimental Psychology: Animal Behavior Processes, 34, 494-500. doi:10.1037/0097-7403.34.4.494

Le Pelley, M. E. (2004). The role of associative history in models of associative learning: A selective review and a hybrid model. Quarterly Journal of Experimental Psychology, 57B, 193-243. doi:10.1080/02724990344000141

Mackintosh, N. J. (1975). A theory of attention: Variations in the associability of stimuli with reinforcement. Psychological Review, 82, 276-298. doi:10.1037/h0076778

McLaren, I. P. L., \& Mackintosh, N. J. (2002). Associative learning and elemental representation: II. Generalization and discrimination. Animal Learning \& Behavior, 30, 177-200.

Pavlov, I. P. (1927). Conditioned reflexes: An investigation of the physiological activity of the cerebral cortex (G. V. Anrep, Trans.). New York: Dover.

Pearce, J. M. (1987). A model for stimulus generalization in Pavlovian conditioning. Psychological Review, 94, 61-73. doi:10.1037/0033 $-295 X .94 .1 .61$

Pearce, J. M. (1994). Similarity and discrimination: A selective review and a connectionist model. Psychological Review, 101, 587-607. doi:10.1037/0033-295X.101.4.587

PeArce, J. M. (2002). Evaluation and development of a connectionist theory of configural learning. Animal Learning \& Behavior, 30, 73-95.

Pearce, J. M., \& Hall, G. (1980). A model for Pavlovian learning: Variations in the effectiveness of conditioned but not of unconditioned stimuli. Psychological Review, 87, 532-552. doi:10.1037/0033 $-295 X .87 .6 .532$

Pearce, J. M., \& Redhead, E. S. (1993). The influence of an irrelevant stimulus on two discriminations. Journal of Experimental Psychol- ogy: Animal Behavior Processes, 19, 180-190. doi:10.1037/0097 -7403.19.2.180

ResCorla, R. A. (1972). "Configural” conditioning in discrete-trial bar pressing. Journal of Comparative \& Physiological Psychology, 79, 307-317. doi: $10.1037 / \mathrm{h} 0032553$

Rescorla, R. A., \& Wagner, A. R. (1972). A theory of Pavlovian conditioning: Variations in the effectiveness of reinforcement and nonreinforcement. In A. H. Black \& W. F. Prokasy (Eds.), Classical conditioning II: Current research and theory (pp. 64-99). New York: Appleton-Century-Crofts.

SPENCE, K. W. (1952). The nature of the response in discrimination learning in animals. Psychological Review, 59, 89-93. doi:10.1037/ h0063067

Thein, T., Westbrook, R. F., \& Harris, J. A. (2008). How the associative strengths of stimuli combine in compound: Summation and overshadowing. Journal of Experimental Psychology: Animal Behavior Processes, 34, 155-166. doi:10.1037/0097-7403.34.1.155

Wagner, A. R. (2003). Context-sensitive elemental theory. Quarterly Journal of Experimental Psychology, 56B, 7-29. doi:10.1080/ 02724990244000133

Wagner, A. R., \& Brandon, S. E. (2001). A componential theory of Pavlovian conditioning. In R. R. Mowrer \& S. B. Klein (Eds.), Handbook of contemporary learning theories (pp. 23-64). Mahwah, NJ: Erlbaum.

Whitlow, J. W., \& WAGNeR, A. R. (1972). Negative patterning in classical conditioning: Summation of response tendencies to isolable and configural components. Psychonomic Science, 27, 299-301.

Williams, D. A., Gawel, J. D., Reimer, D. S., \& Mehta, R. (2005). Resistance to interference in complex negative patterning. Learning \& Behavior, 33, 417-427.

Williams, D. A., Mehta, R., \& Dumont, J.-L. (2004). Conditions favoring superconditioning of irrelevant conditioned stimuli. Journal of Experimental Psychology: Animal Behavior Processes, 30, 148-159. doi:10.1037/0097-7403.30.2.148

(Manuscript received August 7, 2008; revision accepted for publication November 12, 2008.) 